\title{
ARACHIDONIC ACID METABOLITES REGULATE INTERLEUKIN-1 PRODUCTION
}

\author{
S. L. Kunkel and S.W. Chensue \\ Department of Pathology \\ University of Michigan \\ Ann Arbor, Michigan 48109
}

Received March 26, 1985

We have investigated the role of arachidonic acid metabolites in the regulation of interleukin-1 production by murine peritoneal macrophages. Indomethacin a potent inhibitor of prostaglandin synthesis caused a dosedependent augmentation of 1 ipopolysaccharide induced interleukin production (up to 7-fold at $5 \mu \mathrm{M}$ ). In contrast, lipoxygenase inhibitors, nordihydroguarietic acid and nafazatrom had no effect at doses that did not significantiy decrease prostaglandin synthesis. Added to lipopolysaccharide stimulated cultures, PGE, suppressed interleukin in a dose-dependent manner. Zymosan induced interieukin was also augmented by indomethacin but unlike lipopolysaccharide treated cultures was suppressed by nordihydroguarietic acid. These data suggest that arachidonate metabolites may be potent autoregulators of macrophage interleukin-1 production. 01985 Academic Press, Inc.

Interleukin-1 $(I L-1)^{*}$ is being increasingly recognized as a important macrophage/monocyte-derived cytokine with effects on a wide variety of target tissues. Some of these effects include induction of fever (1), lymphocyte proliferation/maturation $(2,3)$, the acute phase response (4), muscle catabolism (5) and fibroblast proliferation (6).

While it is well known that numerous stimuli (e.g. endotoxin, immune complexes, phagocytosis etc) can induce IL-1 production by macrophages $(7,8)$ there is little information regarding the factors which regulate its production. Considering the physiologic importance of this cytokine, especially in inflammatory processes, such information might ultimately aid the therapeutic management of inflammatory disease.

In the present paper we show augmentation of lipopolysaccharide (LPS)induced IL-1 production by the cyclooxygenase inhibitor, indomethacin (INDO)

* Abbreviations used in this paper:

AA, arachidonic acid; IL-1, interleukin-1, INDO, indomethacin; NGDA, nordihydroguarietic acid; LPS, lipopolysaccharide; $C O$, cyclooxygenase; LO, 1 ipoxygenase. 
and direct suppression by exogenously added prostaglandin $\mathrm{PGE}_{2}$. zymosaninduced IL-1 production was also augmented by INDO. Thus, we provide evidence for a model in which cyclooxygenase derived arachidonic acid metabolites act as feedback regulators of IL-1 production.

\section{MATERIALS AND METHODS}

Animals - Female, specific pathogen-free CBA/J mice (Jackson Laboratories, Bar Harbor, ME) were used in all experiments. Mice were maintained under pathogen-free conditions and given food and water ad libitum.

Macrophage cultures - Mouse resident peritoneal macrophages were obtained by peritoneal lavage with sterile RPMI-1640 (Grand Island Biological Co., Grand Island, NY). Cells were washed, counted, then suspended to $1 \times 10^{6} / \mathrm{ml} \mathrm{RPMI}$ with 5\% fetal bovine serum (FBS) and $100 \mathrm{U}$ penicilin and $100 \mu \mathrm{g}$ streptomycin/ml. One $\mathrm{ml}$ of suspension was dispensed onto $35 \mathrm{~mm}$ sterile plastic culture dishes (Corning Glass Works, Corning, NY). After $2 \mathrm{~h}$ incubation, $\left(37^{\circ}\right.$, $5 \% \mathrm{CO}_{2}, 100 \%$ humidity) nonadherent cells were removed by two vigorous rinses.

the monolayers were overlaid with $1 \mathrm{~m} 1$ of serum-free RPMI with antibiotics plus the agent being tested for IL-1 induction, zymosan ( $500 \mu \mathrm{g})$ or Salmonella 1 ipopolysaccharide (10 $\mathrm{\mu g}$ ) (Sigma Chemical Co., St. Louis, MO). Supernates were collected after $18 \mathrm{~h}$ incubation. The total number of macrophages was quantitated by scraping cells from culture dishes and counting in a hemacytometer. These were $>95 \%$ pure by morphology and phagocytic index.

Arachidonic acid metabolites and metabolic inhibitors - Prostaglandins were kindly provided by Dr. J. Pike (Upjohn Co., Kalamazoo, Mich.). Purity of those compounds was checked by high pressure liquid chromatography performed as previously described (9).

Indomethacin and nordihydroguariatic acid were purchased from Sigma. Nafazatrom was kindly provided by Miles Laboratories, Elkhart, Ind. The in vitro specificity of these compounds in the inhibition of cyclooxygenase and 1ipoxygenase pathway has been previously published (10).

Radioimmunoassay for prostaglandins - The macrophage culture medium was collected, centrifuged at $500 \mathrm{xg}$ for $10 \mathrm{~min}$ and then subjected to radioimmunoassay for $\mathrm{PGE}_{2}$ and 6-keto $P G F_{1 \alpha}$ as described by Fitzpatrick et al. $(11,12)$. Briefly, samples were extracted to remove protein and free fatty acids before addition of antibodies. Bound and free ligand were separated using dextrancoated charcoal. Sensitivity for $\mathrm{PGE}_{2}$ and 6 -keto $P G F_{1 \alpha}$ were 8 and 4 picograms, respectiveiy.

Interleukin-1 assay - Interleukin-1 was measured by the standard thymocyte proliferation assay. Briefly, $5 \times 10^{5}$ mouse thymocytes in $0.1 \mathrm{ml}$ RPMI-10\% FBS containing $5 \mu \mathrm{g} / \mathrm{ml}$ PHA (Burroughs Welcome, Research Triangle, NC) were distributed into each well of a 96-well sterile culture dish (Costar, Cambridge, Mass.). Equal volumes of serially diluted test supernate were then added to triplicate wells. Control wells contained no PHA. At $66 \mathrm{~h}$ of incubation, the cells were pulsed with $0.5 \mu \mathrm{Ci}$ of tritiated thymidine then harvested at $72 \mathrm{~h}$. Total thymidine incorporation was determined by liquid scintillation spectrophotometry. Augmentation of PHA-induced thymocyte proliferation indicated IL-1 activity.

Statistical analysis - The Student's t-test was used to compare control and experimental groups, Values of $p>.05$ was considered not significant.

\section{RESULTS}

Effect of inhibitors of arachidonic acid metabolism on lipopolysaccharideinduced interleukin-1 production. Initially, we compared known inhibitors of cyclooxygenase (CO) and lipoxygenase (LO) pathways of AA metabolism for their 

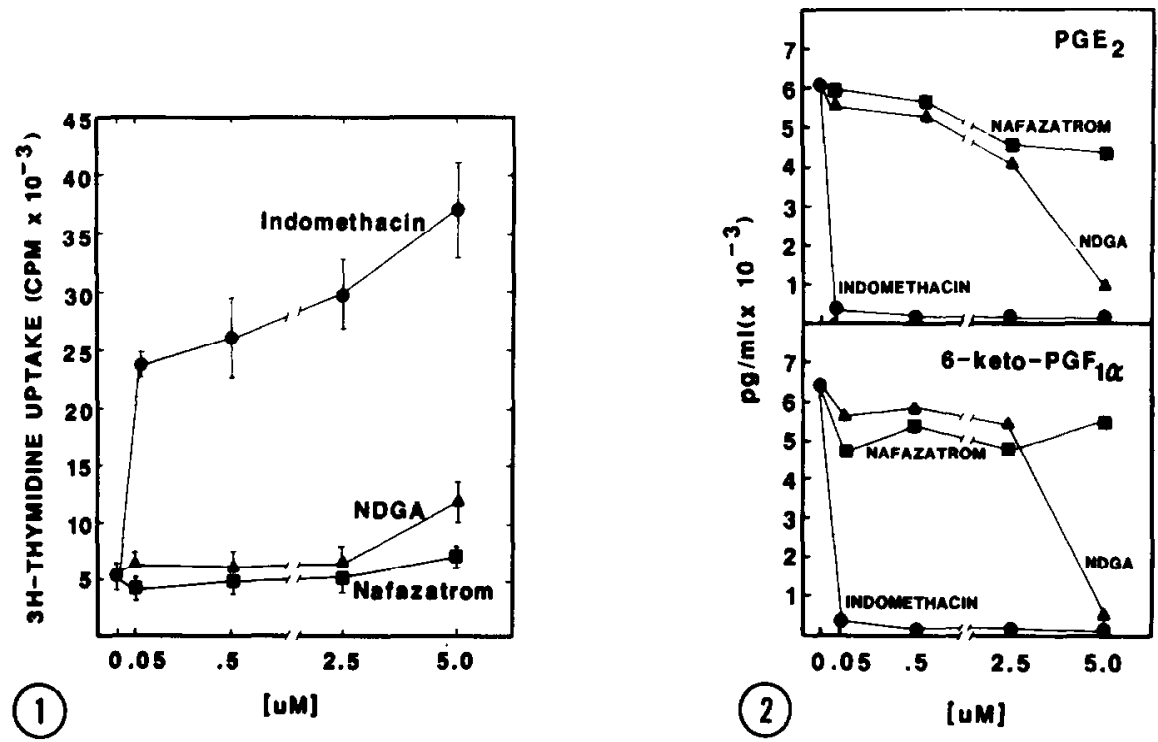

Figure 1. Effect of inhibitors of arachidonic acid metabolism on LPSinduced IL-1 production. Macrophage cultures $\left(2.2 \times 10^{5} /\right.$ dish $)$ were stimulated $18 \mathrm{~h}$ with LPS $(10 \mu \mathrm{g} / \mathrm{ml})$ in the presence of graded concentrations of inhibitors. Supernates were then tested for IL-1 activity. Control macrophage cultures not stimulated with LPS gave the

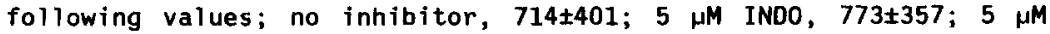
NDGA 986 $\pm 286 ; 5 \mu M$ NAFAZ $699 \pm 289$. Points are mean CPM \pm SD of triplicate determinations.

Figure 2. Levels of prostaglandins in LPS-stimulated macrophage cultures treated with various inhibitors of arachidonic acid metabolism. The culture supernates described in figure 1 were tested by radioimmunoassay for $\mathrm{PGE}_{2}$ and 6-keto-PGF, a spontaneous breakdown product of $\mathrm{PGI}_{2}$ (prostacyclin). Points are mean of three determinations.

effect on LPS-induced IL-1 production. Figure 1 shows the dose-response kinetics of these compounds. A dose-dependent augmentation of IL-1 activity was obtained with the CO inhibitor, INDO, which caused a 7-fold increase at the $5 \mu M$ concentration. In contrast, the LO inhibitors NDGA and nafazatrom had no significant effect over most of the dose range. However, a 2-fold augmentation was caused by NDGA at $5 \mu \mathrm{M}$. Interestingly, the augmentation of IL-1 activity

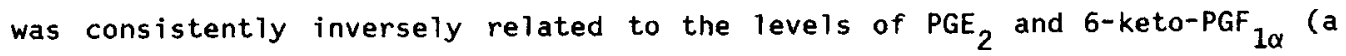
breakdown product of prostacyclin) in the macrophage cultures (Figure 2).

Effect of exogenous added $P_{G}$ on interleukin-1 production. We next determined IL-1 production in PGE $_{2}$ treated LPS-stimulated macrophage cultures. As shown in Figure 3 ( $A$ \& B, closed circles) a dose dependent suppression was observed. Moreover, the augmentation caused by INDO could be overcome by 

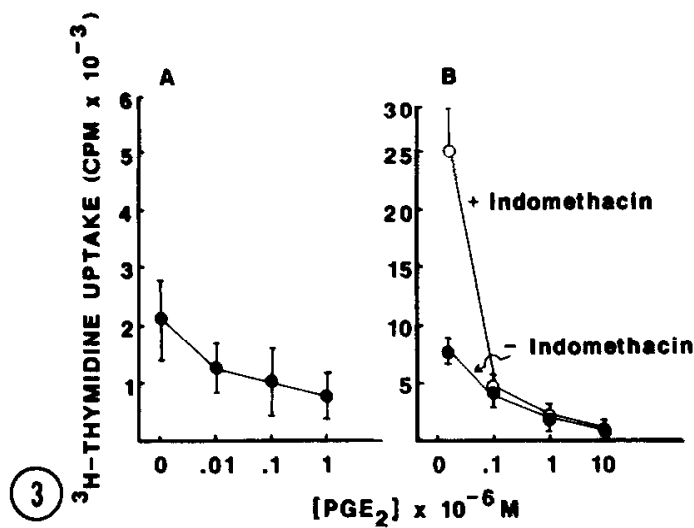

Figure 3.

Effect of exogenous PGE, on LPS-induced IL-1 production. Macrophage cultures were stimutated $18 \mathrm{~h}$ with LPS $(10 \mu \mathrm{g} / \mathrm{m} 1)$ in the presence of graded concentrations of $P G E_{2}$, then supernates were tested for IL-1 activity. A. Suppression by $\mathrm{PGE}_{2}$, unstimulated control 114 132 . B. Suppression by $\mathrm{PGE}_{2}$ in presence or absence of indomethacin $(2.5 \mu \mathrm{M})$, unstimulated control 398 172 . Points are mean $\mathrm{cPm} \pm \mathrm{SD}$ of triplicate determinations.

Figure 4. Effect of arachidonic acid metabolic inhibitors on zymosaninduced IL-1 production. Percent change in IL-1 activity ( 3 H-thymidine uptake) produced by zymosan-stimulated macrophages in the presence of graded concentrations of indomethacin or NDGA. Points are mean $\mathscr{x} \pm$ SE of 3-5 experiments.

exogenous $\mathrm{PGE}_{2}$ treatment (Figure $3 \mathrm{~B}$, open circles). Thus, $\mathrm{PGE}_{2}$ could directly suppress IL-I production.

\section{Effect of inhibitors of arachidonic acid metabolism on zymosan-induced}

interleukin-1 production. When we tested $C O$ and $L O$ inhibitors for their effect on zymosan-induced IL-1 we again saw augmentation by INDO at $2.5 \mu \mathrm{M}$ (Figure 4 ). However, this effect was less dramatic than when LPS was the stimulant. Furthermore, NDGA caused a definite suppression of zymosan-induced IL-1 at 2.5 $\mu M$, an effect not observed when LPS was the stimulant. These data suggested that both $\mathrm{CO}$ and LO-derived metabolites may $\mathrm{play}$ a role in zymosan-induced IL-I synthesis.

\section{DISCUSSION}

It is well established that macrophages produce significant amounts of arachidonate metabolites as well as being a major source of IL-1 (13-15). However, the relationship between these products is not understood. In the present report we provide direct and indirect evidence that products of the co 
pathway $\left(\mathrm{PGE}_{2}, \mathrm{PGI}_{2}\right.$ ) down-regulate the production of $\mathrm{IL}-1$ by macrophages. Thus IL-I synthesis may be profoundly affected by drugs which inhibit arachidonic acid metabolism (e.g. indomethacin).

Our data imply a model of feedback regulation of IL-1 by prostaglandins. Interestingly, IL- 1 can stimulate $\mathrm{PGE}_{2}$ synthesis in target tissues such as the hypothalamus and muscle $(1,5)$. Hence, it demonstrates the classic characteristic of a hormone by inducing its own inhibitor. It is not clear as yet if $I L-1$ plays a role in the induction of $P G$ synthesis by macrophages, however studies to resolve this question are currently underway in our laboratory. The autoregulatory effect of $P G$ on $I L-1$ production is supported by the finding of Boraschi et al (16) showing that gamma-interferon can augment macrophage IL-I production by suppression $\mathrm{PGE}_{2}$ release.

Our finding that IL-1 production was more dramatically augmented by INOO in LPS-stimulated rather than zymosan-stimulated macrophages is most likely related to the differing spectra of $A A$ metabolites induced by these stimuli. Stimulation with membrane perturbants such as LPS causes macrophages to release large amounts of essentially only co products, $P G E_{2}$ and $P G I_{2}$, whereas zymosan stimulates production of lesser amounts of PGs as well as some leukotriene $C_{4}$ $\left(\operatorname{LTC}_{4}\right)(17,18)$. We have confirmed this by high pressure liquid chromatographic analysis of our culture supernates. Furthermore, zymosan is not as potent as LPS in stimulating IL-1 production. Thus, zymosan-stimulated macrophages may be under less tonic suppression by PGs. Moreover, we have recently reported that LTC $_{4}$ can directly stimulate IL-1 production (19) and thus may oppose the suppressive effect of PGs.

The potential stimulatory effect of $\mathrm{LTC}_{4}$ can also explain the observed suppressive effect of NDGA on zymosan-induced IL-1. The synthes is of LTC 4 , a lipoxygenase product, is blocked by NDGA. Hence, this would leave the suppressive action of $P G$ unopposed as well as eliminate a stimulus of $I L-1$ production. It is noteworthy, that Dinarel10 et a1. (2) have reported suppression of human monocyte IL-I production (induced by a particulate stimulus) by lipoxygenase inhibitors, suggesting these mechanisms may also apply to humans. 
In conclusion, we provide evidence that $I L-1$ production by macrophages is autoregulated by arachidonic acid metabolites i.e. suppressed by PGs. The degree of modulation appears to be affected by the nature of the inducing stimulus and possibly by presence of opposing stimuli such as lipoxygenase pathway metabolites.

\section{ACKNOWLEDGEMENT}

This work was supported by NIH grants HL 31237 and HL 31963. The authors wish to express their appreciation for the expert secretarial support of Jeny Breakstone.

\section{REFERENCES}

1. Dinare110, C.A. (1982). Am. J. Med. 72, 799-819.

2. Gery, I. and Waksman, B.H. (1972). J. Exp. Med. 136, 143-155.

3. Lipsky, P.E., Thompson, P.A., Rosenwasser, L.J. and Dinare110, C.A. (1983). J. Immuno]. 130, 2708-2714.

4. Sztein, M.B., Vogel, S.N., Sipe, J.D., Murphy, P.A., Mize1, S.B., Oppenheim, J.J. and Rosenstreich, D.L. (1981). Cell. Immunol. 63, 164-176.

5. Baracos, V., Rodemann, H.P., Dinarello, C.A. and Goldberg, A. L. (1983). N. Eng1. J. Med. 308, 553-558.

6. Schmidt, J.A., Mizel, S.B., Cohen, D. and Green, I. (1982). J. Immunol. $128,2177-2182$.

7. Kampschmidt, R.F. (1984). J. Leuk. Biol. 36, 341-355.

8. Dinarello, C.A. (1984). N. Engl. J. Med. 311, 1413-1418.

9. Chensue, S.W., Kunkel, S.L., Higashi, G.I., Ward, P.A. and Boros, D.L. (1983). Inf. Immun. 42, 1116-1125.

10. Kunke1, S. L., Chensue, S.W., Mouton, C. and Higashi, G. I. (1984). J. C1in. Invest. 74, 514-524.

11. Fitzpatrick, F.A. and Bundy, G.L. (1978). Proc. Nat1. Acad. Sci. USA 75, 2689-2693.

12. Fitzpatrick, F.A. and Wynalda, W.A. (1976). Anal. Biochem. 73, 198-208.

13. Chensue, S.W. and Kunke1, S.L. (1983). C1in. Lab. Med. 3, 677-694.

14. Oppenheim, J.J., Stadler, B.M., Siraganian, M., Mage, M. and Matheison, B. (1982). Fed. Proc. 41, 257-262.

15. Mayernik, D.G., Hag, A. and Rinehart, J.J. (1984). J. Leuk. Biol. 36, 551-557.

16. Boraschi, P., Censini, S. and Tagliabue, A. (1984). J. Immuno]. 133, 764-768.

17. Kurland, J. I. and Bockman, R. (1978). J. Exp. Med. 147, 952-957.

18. Hseuh, W., Desai, U., Gonzalez-Cross, F., Lamb, R. and Chu, A. (1981). Nature 290, 710-713.

19. Chensue, S.W. and Kunke], S. L. (1985). Fed. Proc. 44, 1270.

20. Dinarello, C.A., Bishai, I., Rosenwasser, L.J. and Coceani, F. (1984). Int. J. Immunopharmac. 6, 43-50. 